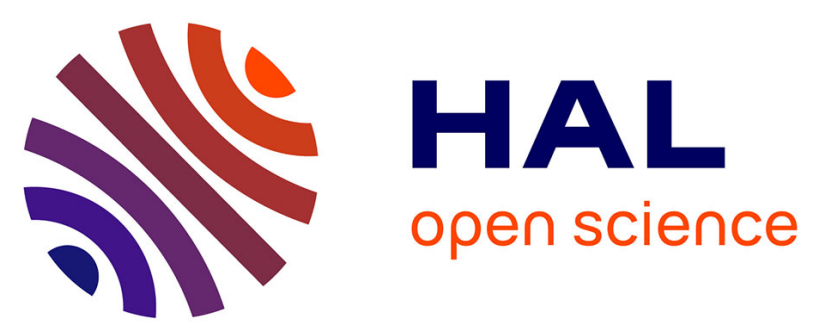

\title{
SOME PROPERTIES OF SEVERAL FUNCTIONS INVOLVING POLYGAMMA FUNCTIONS AND ORIGINATING FROM THE SECTIONAL CURVATURE OF THE BETA MANIFOLD
}

Feng Qi

\section{To cite this version:}

Feng Qi. SOME PROPERTIES OF SEVERAL FUNCTIONS INVOLVING POLYGAMMA FUNCTIONS AND ORIGINATING FROM THE SECTIONAL CURVATURE OF THE BETA MANIFOLD. São Paulo Journal of Mathematical Sciences, 2020, 14 (2), pp.614-630. 10.1007/s40863-02000193-1 . hal-02496404v2

\author{
HAL Id: hal-02496404 \\ https://hal.science/hal-02496404v2
}

Submitted on 23 Nov 2020

HAL is a multi-disciplinary open access archive for the deposit and dissemination of scientific research documents, whether they are published or not. The documents may come from teaching and research institutions in France or abroad, or from public or private research centers.
L'archive ouverte pluridisciplinaire HAL, est destinée au dépôt et à la diffusion de documents scientifiques de niveau recherche, publiés ou non, émanant des établissements d'enseignement et de recherche français ou étrangers, des laboratoires publics ou privés.

\section{(ㄷ)(1) $\$$}

Distributed under a Creative Commons Attribution - NonCommerciall 4.0 International 


\section{Some properties of several functions involving polygamma functions and originating from the sectional curvature of the beta manifold}

Feng Qi

Received: 13 May 2020 / Accepted: 2 October 2020

Please cite this article as "Feng Qi, Some properties of several functions involving polygamma functions and originating from the sectional curvature of the beta manifold, São Paulo Journal of Mathematical Sciences 14 (2020), no. 2, 614-630; available online at https://doi.org/10. 1007/s40863-020-00193-1"

\section{Contents}

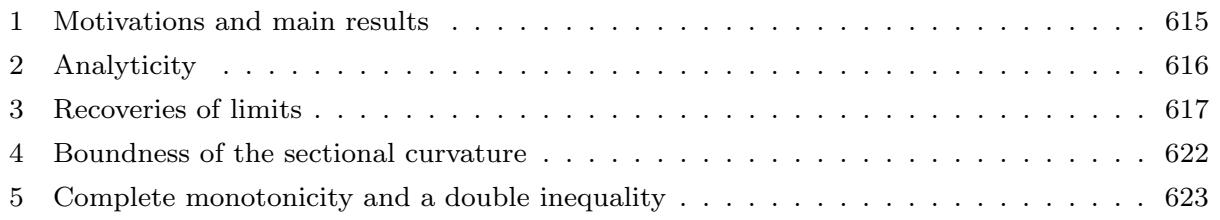

Abstract In the paper, the author investigates some properties, including analyticity, limits, monotonicity, complete monotonicity, and inequalities, of several functions involving the tri-, tetra-, and penta-gamma functions and originating from computation of the sectional curvature of the beta manifold.

Keywords property · polygamma function · analyticity · monotonicity · complete monotonicity · limit · inequality · trigamma function · tetragamma function . pentagamma function $\cdot$ sectional curvature $\cdot$ beta manifold

Mathematics Subject Classification (2010) 26A48 26 A51 33 B15 $\cdot 44$ A 10

\section{Feng Qi}

College of Mathematics and Physics, Inner Mongolia University for Nationalities, Tongliao 028043, Inner Mongolia, China

Institute of Mathematics, Henan Polytechnic University, Jiaozuo 454010, Henan, China School of Mathematical Sciences, Tianjin Polytechnic University, Tianjin 300387, China E-mail: qifeng618@gmail.comm, qifeng618@hotmail.com, qifeng618@qq.com URL: https://qifeng618. wordpress.com 


\section{Motivations and main results}

Let $M=\{(x, y): x, y>0\}$ denote the first quadrant on $\mathbb{R}^{2}$. Let

$$
\mathrm{d} s^{2}=\psi^{\prime}(x) \mathrm{d} x^{2}+\psi^{\prime}(y) \mathrm{d} y^{2}-\psi^{\prime}(x+y)(\mathrm{d} x+\mathrm{d} y)^{2}
$$

be the Fisher metric and $M$ be equipped with $\mathrm{d} s^{2}$, where

$$
\psi(z)=[\ln \Gamma(z)]^{\prime}=\frac{\Gamma^{\prime}(z)}{\Gamma(z)} \quad \text { and } \quad \Gamma(z)=\int_{0}^{\infty} t^{z-1} e^{-t} \mathrm{~d} t
$$

for $\Re(z)>0$. In the literature [1, Section 6.4], the function $\Gamma(z)$ is known as the Euler gamma function, the functions $\psi(z), \psi^{\prime}(z), \psi^{\prime \prime}(z), \psi^{\prime \prime \prime}(z)$, and $\psi^{(4)}(z)$ are known as the di-, tri-, tetra-, penta-, and hexa-gamma functions respectively, and, as a whole, all the derivatives $\psi^{(k)}(z)$ for $k \geq 0$ are known as the polygamma functions.

Proposition 3 in [8, Section 2.4] and Proposition 13 in [9] read that the sectional curvature $K(x, y)$ of the Fisher metric $\mathrm{d} s^{2}$ on the beta manifold $\left(M, \mathrm{~d} s^{2}\right)$ is given by

$$
K(x, y)=\frac{1}{4} \frac{\psi^{\prime \prime}(x) \psi^{\prime \prime}(y) \psi^{\prime \prime}(x+y)\left[\frac{\psi^{\prime}(x)}{\psi^{\prime \prime}(x)}+\frac{\psi^{\prime}(y)}{\psi^{\prime \prime}(y)}-\frac{\psi^{\prime}(x+y)}{\psi^{\prime \prime}(x+y)}\right]}{\left[\psi^{\prime}(x) \psi^{\prime}(x+y)+\psi^{\prime}(y) \psi^{\prime}(x+y)-\psi^{\prime}(x) \psi^{\prime}(y)\right]^{2}} .
$$

Proposition 4 in [8, Section 2.4] and Proposition 14 in [9] state that the asymptotic behavior of the sectional curvature $K(x, y)$ is given by

$$
\begin{gathered}
\lim _{y \rightarrow 0^{+}} K(x, y)=\lim _{y \rightarrow 0^{+}} K(y, x)=\frac{1}{2}\left(\frac{3}{2}-\frac{\psi^{\prime}(x) \psi^{\prime \prime \prime}(x)}{\left[\psi^{\prime \prime}(x)\right]^{2}}\right), \\
\lim _{y \rightarrow \infty} K(x, y)=\lim _{y \rightarrow \infty} K(y, x)=\frac{\psi^{\prime}(x)+x \psi^{\prime \prime}(x)}{4\left[x \psi^{\prime}(x)-1\right]^{2}}, \\
\lim _{(x, y) \rightarrow\left(0^{+}, 0^{+}\right)} K(x, y)=0, \quad \lim _{(x, y) \rightarrow(\infty, \infty)} K(x, y)=-\frac{1}{2}, \\
\lim _{(x, y) \rightarrow\left(0^{+}, \infty\right)} K(x, y)=\lim _{(x, y) \rightarrow\left(\infty, 0^{+}\right)} K(x, y)=-\frac{1}{4} .
\end{gathered}
$$

Recall from [12, Chapter XIII], 25, Chapter 1], and [26, Chapter IV] that, if a function $h(t)$ on an interval $I$ has derivatives of all orders on $I$ and $(-1)^{n} h^{(n)}(t) \geq 0$ for $t \in I$ and $n \in\{0\} \cup \mathbb{N}$, then we call $h(t)$ a completely monotonic function on $I$. Theorem $12 \mathrm{~b}$ in $[26$, p. 161] states that a necessary and sufficient condition for $h(t)$ to be completely monotonic on the infinite interval $(0, \infty)$ is that

$$
h(t)=\int_{0}^{\infty} e^{-t s} \mathrm{~d} \sigma(s), \quad t \in(0, \infty),
$$

where $\sigma(s)$ is non-decreasing and the integral in 1.6$)$ converges for $t \in(0, \infty)$. The integral representation 1.6 means that a function $h(t)$ is completely monotonic 
on the infinite interval $(0, \infty)$ if and only if it is a Laplace transform of a nondecreasing measure $\sigma(s)$ on the infinite interval $(0, \infty)$.

In this paper, we will show analyticity of the sectional curvature $K(x, y)$, recover the above limits in $1.2,(1.3), 1.4$, and 1.5 by alternative approaches, present that the function

$$
\mathcal{H}(x)=\frac{1}{2}\left(\frac{3}{2}-\frac{\psi^{\prime}(x) \psi^{\prime \prime \prime}(x)}{\left[\psi^{\prime \prime}(x)\right]^{2}}\right)
$$

in 1.2 is decreasing from $(0, \infty)$ onto $\left(-\frac{1}{4}, 0\right)$, reveal necessary and sufficient conditions on $\alpha$ for the function

$$
\mathfrak{H}_{\alpha}(x)=\psi^{\prime}(x)+x \psi^{\prime \prime}(x)+\alpha\left[x \psi^{\prime}(x)-1\right]^{2}
$$

and its negativity to be completely monotonic on $(0, \infty)$, and derive a sharp double inequality

$$
-2<\frac{\psi^{\prime}(x)+x \psi^{\prime \prime}(x)}{\left[x \psi^{\prime}(x)-1\right]^{2}}<-1
$$

in the sense that the constants -2 and -1 cannot be replaced by any bigger and smaller ones respectively.

\section{Analyticity}

In this section, we show analyticity of the sectional curvature $K(x, y)$ on the first quadrant $M$.

Theorem 1 The sectional curvature $K(x, y)$ is an analytic function of $(x, y)$ on the first quadrant $M$.

Proof Since

$$
\psi^{(n)}(z)=(-1)^{n+1} \int_{0}^{\infty} \frac{t^{n}}{1-e^{-t}} e^{-z t} \mathrm{~d} t
$$

for $\Re(z)>0$ and $n \geq 1$, see [1, p. 260, 6.4.1], the trigamma function $\psi^{\prime}(x)$ is positive, decreasing, and convex on $(0, \infty)$, with the $\operatorname{limits}_{x \rightarrow 0^{+}} \psi^{\prime}(x)=\infty$ and $\lim _{x \rightarrow \infty} \psi^{\prime}(x)=0$. Applying the double inequality

$$
\frac{n-1}{n}<\frac{\left[\psi^{(n)}(x)\right]^{2}}{\psi^{(n-1)}(x) \psi^{(n+1)}(x)}<\frac{n}{n+1}
$$

for $n \geq 2$, see [4, Corollary 2.3], [18, Section 3.5], or 27, Eq. (1.4)], leads to

$$
\left[\frac{1}{\psi^{\prime}(x)}\right]^{\prime \prime}=\frac{2\left[\psi^{\prime \prime}(x)\right]^{2}-\psi^{\prime}(x) \psi^{\prime \prime \prime}(x)}{\left[\psi^{\prime}(x)\right]^{3}}>0 \text {. }
$$


A function $\varphi(x)$ is said to be sub-additive on an interval $I$ if $\varphi(x+y)<\varphi(x)+$ $\varphi(y)$ holds for all $x, y \in I$ with $x+y \in I$. If $\varphi(x+y)>\varphi(x)+\varphi(y)$, then the function $\varphi(x)$ is called super-additive on the interval $I$. A function $\varphi:[0, \infty) \rightarrow$ $\mathbb{R}$ is said to be star-shaped if $\varphi(\nu t)<\nu \varphi(t)$ for $\nu \in[0,1]$ and $t \geq 0$. Between convex functions, star-shaped functions, and super-additive functions, there are the following relations:

1. if $\varphi$ is convex on $[0, \infty)$ with $\varphi(0) \leq 0$, then $\varphi$ is star-shaped;

2. if $\varphi:[0, \infty) \rightarrow \mathbb{R}$ is star-shaped, then $\varphi$ is super-additive.

For more information on additive and star-shaped functions, please refer to 11 , Chapter 16], [13, Section 3.4], the papers [2,3, 5, 7, 10,22, 23], and closely related references therein. By these relations, we conclude that the reciprocal $\frac{1}{\psi^{\prime}(x)}$ is super-additive. Hence, the factor of the denominator in (1.1), which can be rearranged as

$$
\begin{aligned}
& \psi^{\prime}(x) \psi^{\prime}(x+y)+\psi^{\prime}(y) \psi^{\prime}(x+y)-\psi^{\prime}(x) \psi^{\prime}(y) \\
& =\psi^{\prime}(x) \psi^{\prime}(y) \psi^{\prime}(x+y)\left[\frac{1}{\psi^{\prime}(x)}+\frac{1}{\psi^{\prime}(y)}-\frac{1}{\psi^{\prime}(x+y)}\right]>0,
\end{aligned}
$$

is positive on $(0, \infty)$.

Since $\psi^{(n)}(z)$ for $n \geq 0$ is a single valued analytic function over the entire complex plane save at the points $z=-m$ where it possesses poles of order $n+1$, see [1. p. 260, 6.4.1] or the paper [16, the denominator in (1.1) is positive and analytic on $(0, \infty)$. The numerator in 1.1 is also analytic on $(0, \infty)$. In conclusion, the sectional curvature $K(x, y)$ is analytic on the first quadrant $M$. The proof of Theorem 1 is complete.

\section{Recoveries of limits}

In this section, we recover the limits in $(1.2), 1.3,1.4$, and 1.5 by alternative approaches.

Theorem 2 The sectional curvature $K(x, y)$ on the first quadrant $M$ has the limits

$$
\begin{aligned}
\lim _{x \rightarrow \infty} K(x, y) & =\frac{\psi^{\prime}(y)+y \psi^{\prime \prime}(y)}{4\left[1-y \psi^{\prime}(y)\right]^{2}}, \\
\lim _{y \rightarrow \infty} K(x, y) & =\frac{\psi^{\prime}(x)+x \psi^{\prime \prime}(x)}{4\left[x \psi^{\prime}(x)-1\right]^{2}}, \\
\lim _{y \rightarrow 0^{+}} \lim _{x \rightarrow \infty} K(x, y) & =-\frac{1}{4}, \\
\lim _{y \rightarrow \infty} \lim _{x \rightarrow \infty} K(x, y) & =-\frac{1}{2},
\end{aligned}
$$




$$
\begin{aligned}
\lim _{x \rightarrow 0^{+}} \lim _{y \rightarrow \infty} K(x, y) & =-\frac{1}{4}, \\
\lim _{x \rightarrow \infty} \lim _{y \rightarrow \infty} K(x, y) & =-\frac{1}{2}, \\
\lim _{(x, y) \rightarrow(\infty, \infty)} K(x, y) & =-\frac{1}{2} .
\end{aligned}
$$

Proof The expression (1.1) for the sectional curvature $K(x, y)$ can be rewritten as

$$
K(x, y)=\frac{\left(\begin{array}{c}
{\left[x^{2} \psi^{\prime \prime}(x)\right] \psi^{\prime \prime}(y)\left[(x+y)^{2} \psi^{\prime \prime}(x+y)\right]} \\
\times\left[\frac{\psi^{\prime}(x)}{\psi^{\prime \prime}(x)}-\frac{\psi^{\prime}(x+y)}{\psi^{\prime \prime}(x+y)}\right] \\
+\left[x^{2} \psi^{\prime \prime}(x)\right] \psi^{\prime}(y)\left[(x+y)^{2} \psi^{\prime \prime}(x+y)\right]
\end{array}\right)}{4\left(\begin{array}{c}
\left.\psi^{\prime}(y) x(x+y)\left[\psi^{\prime}(x+y)-\psi^{\prime}(x)\right]\right)^{2} \\
+\left[x \psi^{\prime}(x)\right]\left[(x+y) \psi^{\prime}(x+y)\right]
\end{array}\right)} .
$$

Making use of the limit

$$
\lim _{x \rightarrow \infty}\left[x^{k} \psi^{(k)}(x)\right]=(-1)^{k-1}(k-1) !, \quad k \geq 1
$$

in 6, p. 9896, Eq. (13)], 19, p. 1689, Eq. (3.3)], 20, p. 286, Eq. (2.6)], and [21, p. 81, Eq. (41)], we can obtain

$$
\lim _{x \rightarrow \infty}\left[x^{2} \psi^{\prime \prime}(x)\right]=\lim _{x \rightarrow \infty}\left[(x+y)^{2} \psi^{\prime \prime}(x+y)\right]=-1
$$

and

$$
\lim _{x \rightarrow \infty}\left[x \psi^{\prime}(x)\right]=\lim _{x \rightarrow \infty}\left[(x+y) \psi^{\prime}(x+y)\right]=1 .
$$

Direct differentiating and utilizing the limit 3.9 lead to

$$
\left[\frac{\psi^{\prime}(x)}{\psi^{\prime \prime}(x)}\right]^{\prime}=1-\frac{\left[x \psi^{\prime}(x)\right]\left[x^{3} \psi^{\prime \prime \prime}(x)\right]}{\left[x^{2} \psi^{\prime \prime}(x)\right]^{2}} \rightarrow 1-\frac{0 ! 2 !}{(1 !)^{2}}=-1
$$

as $x \rightarrow \infty$. Therefore, by virtue of Lagrange's mean value theorem, we have

$$
\lim _{x \rightarrow \infty}\left[\frac{\psi^{\prime}(x)}{\psi^{\prime \prime}(x)}-\frac{\psi^{\prime}(x+y)}{\psi^{\prime \prime}(x+y)}\right]=-y \lim _{x \rightarrow \infty}\left[\frac{\psi^{\prime}(t)}{\psi^{\prime \prime}(t)}\right]_{t=\xi}^{\prime}=y
$$

where $\xi \in(x, x+y)$.

Employing Lagrange's mean value theorem, simple differentiating, and utilizing the limit 3.9 yield

$$
x(x+y)\left[\psi^{\prime}(x+y)-\psi^{\prime}(x)\right]=x\left[(x+y) \psi^{\prime}(x+y)-x \psi^{\prime}(x)\right]-y\left[x \psi^{\prime}(x)\right]
$$




$$
\begin{aligned}
& =x\left[\psi^{\prime}(\xi)+\xi \psi^{\prime \prime}(\xi)\right]-y\left[x \psi^{\prime}(x)\right], \quad \xi \in(x, x+y) \\
& =\frac{x}{\xi}\left[\xi \psi^{\prime}(\xi)+\xi^{2} \psi^{\prime \prime}(\xi)\right]-y\left[x \psi^{\prime}(x)\right], \quad \xi \in(x, x+y) \\
& \rightarrow-y, \quad x, \xi \rightarrow \infty
\end{aligned}
$$

Substituting the limits in $3.10,3.11,3.12$, and 3.13 into 3.8 arrives at the limit (3.1).

For $\Re(z)>0$ and $k \geq 1$, we have

$$
\psi^{(k-1)}(z+1)=\psi^{(k-1)}(z)+(-1)^{k-1} \frac{(k-1) !}{z^{k}} .
$$

See 1. p. 260, 6.4.6]. From this, it follows that

$$
\begin{aligned}
\lim _{x \rightarrow 0^{+}}\left[x^{k} \psi^{(k-1)}(x)\right] & =\lim _{x \rightarrow 0^{+}}\left\{x^{k}\left[\psi^{(k-1)}(x+1)-(-1)^{k-1} \frac{(k-1) !}{x^{k}}\right]\right\} \\
& =(-1)^{k}(k-1) !
\end{aligned}
$$

for $k \geq 1$. See [15, p. 260, (2.2)] or [27, p. 769]. From the limits (3.1) and (3.15), it follows that

$$
\lim _{y \rightarrow 0^{+}} \lim _{x \rightarrow \infty} K(x, y)=\lim _{y \rightarrow 0^{+}} \frac{y^{2} \psi^{\prime}(y)+y^{3} \psi^{\prime \prime}(y)}{4\left[y-y^{2} \psi^{\prime}(y)\right]^{2}}=\frac{1 !-2 !}{4(0-1 !)}=-\frac{1}{4} .
$$

The limit 3.3 is thus proved.

In [1, p. 260, 6.4.11], it was given that, for $|\arg z|<\pi$, as $z \rightarrow \infty$,

$$
\psi^{(n)}(z) \sim(-1)^{n-1}\left[\frac{(n-1) !}{z^{n}}+\frac{n !}{2 z^{n+1}}+\sum_{k=1}^{\infty} B_{2 k} \frac{(2 k+n-1) !}{(2 k) ! z^{2 k+n}}\right],
$$

see also 24], where $B_{2 k}$ for $n \geq 0$ are known as the Bernoulli numbers which can be generated [14] by

$$
\frac{z}{e^{z}-1}=1-\frac{z}{2}+\sum_{k=1}^{\infty} B_{2 k} \frac{z^{2 k}}{(2 k) !}, \quad|z|<2 \pi .
$$

Accordingly, we can derive

$$
\begin{aligned}
\lim _{y \rightarrow \infty}\left(y^{2}\left[\psi^{\prime}(y)+y \psi^{\prime \prime}(y)\right]\right)= & \lim _{y \rightarrow \infty}\left\{y ^ { 2 } \left[\frac{1}{y}+\frac{1}{2 y^{2}}+\sum_{k=1}^{\infty} B_{2 k} \frac{(2 k) !}{(2 k) ! y^{2 k+1}}\right.\right. \\
& \left.\left.-y\left(\frac{1}{y^{2}}+\frac{1}{y^{3}}+\sum_{k=1}^{\infty} B_{2 k} \frac{(2 k+1) !}{(2 k) ! y^{2 k+2}}\right)\right]\right\} \\
= & \lim _{y \rightarrow \infty}\left[-\frac{1}{2}+\sum_{k=1}^{\infty} B_{2 k} \frac{(2 k) !-(2 k+1) !}{(2 k) ! y^{2 k-1}}\right]
\end{aligned}
$$




$$
=-\frac{1}{2}
$$

and

$$
\begin{aligned}
\lim _{y \rightarrow \infty}\left(y\left[1-y \psi^{\prime}(y)\right]\right) & =\lim _{y \rightarrow \infty}\left\{y\left[1-y\left(\frac{1}{y}+\frac{1}{2 y^{2}}+\sum_{k=1}^{\infty} B_{2 k} \frac{(2 k) !}{(2 k) ! y^{2 k+1}}\right)\right]\right\} \\
& =\lim _{y \rightarrow \infty}\left[-\frac{1}{2}-\sum_{k=1}^{\infty} B_{2 k} \frac{(2 k) !}{(2 k) ! y^{2 k-1}}\right] \\
& =-\frac{1}{2}
\end{aligned}
$$

Consequently, it follows that

$$
\begin{aligned}
\lim _{y \rightarrow \infty} \lim _{x \rightarrow \infty} K(x, y) & =\frac{1}{4} \lim _{y \rightarrow \infty} \frac{\psi^{\prime}(y)+y \psi^{\prime \prime}(y)}{\left[1-y \psi^{\prime}(y)\right]^{2}} \\
& =\frac{1}{4} \lim _{y \rightarrow \infty} \frac{y^{2}\left[\psi^{\prime}(y)+y \psi^{\prime \prime}(y)\right]}{y^{2}\left[1-y \psi^{\prime}(y)\right]^{2}} \\
& =\frac{1}{4} \frac{\lim _{y \rightarrow \infty}\left(y^{2}\left[\psi^{\prime}(y)+y \psi^{\prime \prime}(y)\right]\right)}{\lim _{y \rightarrow \infty}\left(y\left[1-y \psi^{\prime}(y)\right]\right)^{2}} \\
& =\frac{1}{4} \frac{-1 / 2}{(-1 / 2)^{2}} \\
& =-\frac{1}{2} .
\end{aligned}
$$

The limit (3.4) is thus proved.

Since $K(x, y)=K(y, x)$, repeating the above arguments acquires the limits 3.2 , 3.5, and (3.6).

Since the sectional curvature $K(x, y)$ is analytic on the first quadrant $M$, see Theorem 1 in this paper, we have

$$
\lim _{(x, y) \rightarrow(\infty, \infty)} K(x, y)=\lim _{x \rightarrow \infty} \lim _{y \rightarrow \infty} K(x, y)=\lim _{y \rightarrow \infty} \lim _{x \rightarrow \infty} K(x, y)=-\frac{1}{2} .
$$

The limit in (3.7) is thus proved. The proof of Theorem 2 is complete.

Theorem 3 The sectional curvature $K(x, y)$ on the first quadrant $M$ has the limits

$$
\begin{aligned}
\lim _{x \rightarrow 0^{+}} K(x, y) & =\frac{1}{2}\left(\frac{3}{2}-\frac{\psi^{\prime}(y) \psi^{\prime \prime \prime}(y)}{\left[\psi^{\prime \prime}(y)\right]^{2}}\right), \\
\lim _{y \rightarrow 0^{+}} K(x, y) & =\frac{1}{2}\left(\frac{3}{2}-\frac{\psi^{\prime}(x) \psi^{\prime \prime \prime}(x)}{\left[\psi^{\prime \prime}(x)\right]^{2}}\right), \\
\lim _{y \rightarrow \infty} \lim _{x \rightarrow 0^{+}} K(x, y) & =-\frac{1}{4}, \\
\lim _{y \rightarrow 0^{+}} \lim _{x \rightarrow 0^{+}} K(x, y) & =0,
\end{aligned}
$$




$$
\begin{aligned}
\lim _{x \rightarrow \infty} \lim _{y \rightarrow 0^{+}} K(x, y) & =-\frac{1}{4}, \\
\lim _{x \rightarrow 0^{+}} \lim _{y \rightarrow 0^{+}} K(x, y) & =0, \\
\lim _{(x, y) \rightarrow\left(0^{+}, 0^{+}\right)} K(x, y) & =0, \\
\lim _{(x, y) \rightarrow\left(0^{+}, \infty\right)} K(x, y) & =-\frac{1}{4}, \\
\lim _{(x, y) \rightarrow\left(\infty, 0^{+}\right)} K(x, y) & =-\frac{1}{4} .
\end{aligned}
$$

Proof By virtue of the L'Hospital rule, we have

$$
\begin{aligned}
\lim _{x \rightarrow 0^{+}} \frac{\frac{\psi^{\prime \prime}(x+y)}{\psi^{\prime}(x+y)}-\frac{\psi^{\prime \prime}(y)}{\psi^{\prime}(y)}}{x} & =\lim _{x \rightarrow 0^{+}} \frac{\mathrm{d}}{\mathrm{d} x}\left[\frac{\psi^{\prime \prime}(x+y)}{\psi^{\prime}(x+y)}\right] \\
& =\lim _{x \rightarrow 0^{+}} \frac{\psi^{\prime}(x+y) \psi^{\prime \prime \prime}(x+y)-\left[\psi^{\prime \prime}(x+y)\right]^{2}}{\left[\psi^{\prime}(x+y)\right]^{2}} \\
& =\frac{\psi^{\prime}(y) \psi^{\prime \prime \prime}(y)-\left[\psi^{\prime \prime}(y)\right]^{2}}{\left[\psi^{\prime}(y)\right]^{2}}
\end{aligned}
$$

and

$$
\lim _{x \rightarrow 0^{+}} \frac{\psi^{\prime}(x+y)-\psi^{\prime}(y)}{x}=\lim _{x \rightarrow 0^{+}} \psi^{\prime \prime}(x+y)=\psi^{\prime \prime}(y)
$$

Using the expression in 1.1 and the limits $3.15,(3.26)$, and 3.27 yields

$$
\begin{aligned}
K(x, y) & =\frac{\left(\begin{array}{c}
{\left[x^{3} \psi^{\prime \prime}(x)\right] \psi^{\prime}(x+y) \psi^{\prime}(y) \frac{\frac{\psi^{\prime \prime}(x+y)}{\psi^{\prime}(x+y)}-\frac{\psi^{\prime \prime}(y)}{\psi^{\prime}(y)}}{+\left[x^{2} \psi^{\prime}(x)\right] \psi^{\prime \prime}(y) \psi^{\prime \prime}(x+y)}}
\end{array}\right)}{4\left(\left[x^{2} \psi^{\prime}(x)\right] \frac{\psi^{\prime}(x+y)-\psi^{\prime}(y)}{x}+x \psi^{\prime}(x+y) \psi^{\prime}(y)\right)^{2}} \\
& \rightarrow \frac{-2 !\left[\psi^{\prime}(y)\right]^{2} \frac{\psi^{\prime}(y) \psi^{\prime \prime \prime}(y)-\left[\psi^{\prime \prime}(y)\right]^{2}}{\left[\psi^{\prime}(y)\right]^{2}}+1 !\left[\psi^{\prime \prime}(y)\right]^{2}}{4\left[1 ! \psi^{\prime \prime}(y)+0\right]^{2}} \\
= & \frac{3\left[\psi^{\prime \prime}(y)\right]^{2}-2 \psi^{\prime}(y) \psi^{\prime \prime \prime}(y)}{4\left[\psi^{\prime \prime}(y)\right]^{2}}
\end{aligned}
$$

as $x \rightarrow 0^{+}$. The limit 3.17 is thus proved.

From the limits 3.9 and 3.17 , it follows that

$$
\lim _{y \rightarrow \infty} \lim _{x \rightarrow 0^{+}} K(x, y)=\frac{3}{4}-\frac{1}{2} \lim _{y \rightarrow \infty} \frac{y \psi^{\prime}(y) y^{3} \psi^{\prime \prime \prime}(y)}{\left[y^{2} \psi^{\prime \prime}(y)\right]^{2}}=\frac{3}{4}-\frac{1}{2} \frac{0 ! 2 !}{(1 !)^{2}}=-\frac{1}{4}
$$

The limit 3.19 is thus proved.

Utilizing the limit 3.15 gives

$$
\frac{3\left[\psi^{\prime \prime}(y)\right]^{2}-2 \psi^{\prime}(y) \psi^{\prime \prime \prime}(y)}{\left[\psi^{\prime \prime}(y)\right]^{2}}=\frac{3\left[y^{3} \psi^{\prime \prime}(y)\right]^{2}-2\left[y^{2} \psi^{\prime}(y)\right]\left[y^{4} \psi^{\prime \prime \prime}(y)\right]}{\left[y^{3} \psi^{\prime \prime}(y)\right]^{2}}
$$




$$
\begin{aligned}
& \rightarrow \frac{3(-2 !)^{2}-2 \times 1 ! \times 3 !}{(-2 !)^{2}} \\
& =0
\end{aligned}
$$

as $y \rightarrow 0^{+}$. This means that the limit 3.20 is valid.

Since $K(x, y)=K(y, x)$, repeating the above proofs of the limits 3.17, 3.19, and 3.20 , we can obtain the limits 3.18, 3.21, and 3.22 immediately.

Combining analyticity of the sectional curvature $K(x, y)$ on $M$, see Theorem 1 in this paper, with the limits 3.20 and 3.22 leads to the limit 3.23 .

Combining analyticity of the sectional curvature $K(x, y)$ on the first quadrant $M$ with the limits $3.3,3.5,3.19$, and 3.21 arrives at the limits 3.24 and (3.25). The proof of Theorem 3 is complete.

\section{Boundness of the sectional curvature}

As did in [8, Proposition 5] and its proof, basing on analyticity and limits recovered in the above two sections, we can deduce that the sectional curvature $K(x, y)$ is negative and bounded on the first quadrant $M$.

Judging from the graph of $K(x, y)$ in Figure 1 and basing on analyticity in

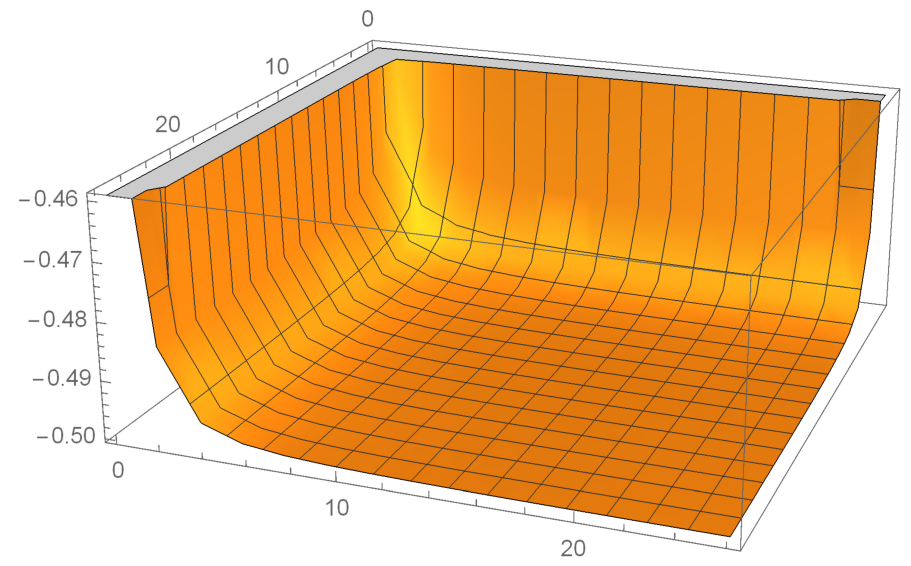

Fig. 1 The lower bound of $K(x, y)$ is conjectured to be $-\frac{1}{2}$

Theorem 1 and the limits in Theorems 2 and 3 , the authors of the papers 8,9 conjectured that $K(x, y)>-\frac{1}{2}$ on $M$. Till 9 September 2020, this conjecture has not been verified and is still open. 


\section{Complete monotonicity and a double inequality}

In the is section, we demonstrate decreasing monotonicity of the function $\mathcal{H}(x)$ in (1.7), find necessary and sufficient conditions for the function $\pm \mathfrak{H}_{\alpha}(x)$ in 1.8 to be completely monotonic on $(0, \infty)$, and derive the double inequality 1.9 .

Theorem 4 The function $\mathcal{H}(x)$ in 1.7 is decreasing from $(0, \infty)$ onto $\left(-\frac{1}{4}, 0\right)$.

The function $\mathfrak{H}_{\alpha}(x)$ in 1.8 is completely monotonic on $(0, \infty)$ if and only if $\alpha \geq 2$, while the function $-\mathfrak{H}_{\alpha}(x)$ is completely monotonic on $(0, \infty)$ if and only if $\alpha \leq 1$.

The double inequality (1.9) is valid and sharp in the sense that the constants -2 and -1 cannot be replaced by any bigger and smaller ones respectively.

Proof The decreasing monotonicity of the function $\mathcal{H}(x)$ in 1.7 follows from the fact that the ratio $\frac{\left[\psi^{\prime \prime}(x)\right]^{2}}{\psi^{\prime}(x) \psi^{\prime \prime \prime}(x)}$ is decreasing from $(0, \infty)$ onto $\left(\frac{1}{2}, \frac{2}{3}\right)$. This fact is a

special case of 27. Theorem 2] which states that the function $\frac{\left[\psi^{(n+1)}(x)\right]^{2}}{\psi^{(n)}(x) \psi^{(n+2)}(x)}$ for $n \geq 1$ is decreasing from $(0, \infty)$ onto $\left(\frac{n}{n+1}, \frac{n+1}{n+2}\right)$.

Making use of the integral representation 2.1 and integrating by parts yield

$$
\begin{gathered}
x \psi^{\prime}(x)=x \int_{0}^{\infty} \frac{t}{1-e^{-t}} e^{-x t} \mathrm{~d} t \\
=-\int_{0}^{\infty} \frac{t}{1-e^{-t}} \frac{\mathrm{d} e^{-x t}}{\mathrm{~d} t} \mathrm{~d} t \\
=-\left[\left.\frac{t e^{-x t}}{1-e^{-t}}\right|_{t \rightarrow 0^{+}} ^{t \rightarrow \infty}-\int_{0}^{\infty}\left(\frac{t}{1-e^{-t}}\right)^{\prime} e^{-x t} \mathrm{~d} t\right] \\
=\int_{0}^{\infty} \frac{e^{t}\left(e^{t}-1-t\right)}{\left(e^{t}-1\right)^{2}} e^{-x t} \mathrm{~d} t+1 \\
\quad>1, \quad \frac{e^{t}\left(e^{t}-1-t\right)}{\left(e^{t}-1\right)^{2}} e^{-x t} \mathrm{~d} t \\
=-\int_{0}^{\infty} \frac{e^{t}\left(e^{t}-1-t\right)}{\left(e^{t}-1\right)^{2}} \frac{\mathrm{d} e^{-x t}}{\mathrm{~d} t} \mathrm{~d} t \\
=-\left[\left.\frac{e^{t}\left(e^{t}-1-t\right) e^{-x t}}{\left(e^{t}-1\right)^{2}}\right|_{t \rightarrow 0^{+}} ^{t \rightarrow \infty}-\int_{0}^{\infty}\left[\frac{e^{t}\left(e^{t}-1-t\right)}{\left(e^{t}-1\right)^{2}}\right]^{\prime} e^{-x t} \mathrm{~d} t\right] \\
=\frac{1}{2}+\int_{0}^{\infty} \frac{e^{t}\left[e^{t}(t-2)+t+2\right]}{\left(e^{t}-1\right)^{3}} e^{-x t} \mathrm{~d} t \\
\rightarrow \frac{1}{2}, \quad x \rightarrow \infty,
\end{gathered}
$$


and

$$
\begin{aligned}
x \psi^{\prime \prime}(x) & =-x \int_{0}^{\infty} \frac{t^{2}}{1-e^{-t}} e^{-x t} \mathrm{~d} t \\
& =\int_{0}^{\infty} \frac{t^{2}}{1-e^{-t}} \frac{\mathrm{d} e^{-x t}}{\mathrm{~d} t} \mathrm{~d} t \\
& =\left[\left.\frac{t^{2} e^{-x t}}{1-e^{-t}}\right|_{t \rightarrow 0^{+}} ^{t \rightarrow \infty}-\int_{0}^{\infty}\left(\frac{t^{2}}{1-e^{-t}}\right)^{\prime} e^{-x t} \mathrm{~d} t\right] \\
& =-\int_{0}^{\infty} \frac{e^{t}\left(2 e^{t}-t-2\right) t}{\left(e^{t}-1\right)^{2}} e^{-x t} \mathrm{~d} t .
\end{aligned}
$$

Therefore, applying (2.1) for $n=1$ and $(5.3)$ gives

$$
\psi^{\prime}(x)+x \psi^{\prime \prime}(x)=-\int_{0}^{\infty} \frac{t\left(e^{t}-1-t\right) e^{t}}{\left(e^{t}-1\right)^{2}} e^{-x t} \mathrm{~d} t<0, \quad x \in(0, \infty) .
$$

If the function $\mathfrak{H}_{\alpha}(x)$ is completely monotonic on $(0, \infty)$, then its first derivative

$$
\mathfrak{H}_{\alpha}^{\prime}(x)=2 \alpha\left[x \psi^{\prime}(x)-1\right]\left[\psi^{\prime}(x)+x \psi^{\prime \prime}(x)\right]+2 \psi^{\prime \prime}(x)+x \psi^{\prime \prime \prime}(x) \leq 0
$$

which is equivalent to

$$
\begin{aligned}
\alpha & \geq-\frac{2 \psi^{\prime \prime}(x)+x \psi^{\prime \prime \prime}(x)}{2\left[x \psi^{\prime}(x)-1\right]\left[\psi^{\prime}(x)+x \psi^{\prime \prime}(x)\right]} \\
& =-\frac{1}{2} \frac{x^{3}\left[2 \psi^{\prime \prime}(x)+x \psi^{\prime \prime \prime}(x)\right]}{\left(x\left[x \psi^{\prime}(x)-1\right]\right)\left(x^{2}\left[\psi^{\prime}(x)+x \psi^{\prime \prime}(x)\right]\right)} \\
& \rightarrow-\frac{1}{2} \frac{\lim _{x \rightarrow \infty}\left(x^{3}\left[2 \psi^{\prime \prime}(x)+x \psi^{\prime \prime \prime}(x)\right]\right)}{\lim _{x \rightarrow \infty}\left(x\left[x \psi^{\prime}(x)-1\right]\right) \lim _{x \rightarrow \infty}\left(x^{2}\left[\psi^{\prime}(x)+x \psi^{\prime \prime}(x)\right]\right)}, \quad x \rightarrow \infty \\
& =-\frac{1}{2} \frac{1}{\frac{1}{2} \times\left(-\frac{1}{2}\right)} \\
& =2
\end{aligned}
$$

where we used (5.1), 5.2), (5.4), and the asymptotic expansion (3.16). Similarly, if the function $-H_{\alpha}(x)$ is completely monotonic on $(0, \infty)$, then

$$
\begin{aligned}
\alpha & \leq-\frac{2 \psi^{\prime \prime}(x)+x \psi^{\prime \prime \prime}(x)}{2\left[x \psi^{\prime}(x)-1\right]\left[\psi^{\prime}(x)+x \psi^{\prime \prime}(x)\right]} \\
& =-\frac{1}{2} \frac{x^{3}\left[2 \psi^{\prime \prime}(x)+x \psi^{\prime \prime \prime}(x)\right]}{\left(x\left[x \psi^{\prime}(x)-1\right]\right)\left(x^{2}\left[\psi^{\prime}(x)+x \psi^{\prime \prime}(x)\right]\right)} \\
& \rightarrow-\frac{1}{2} \frac{\lim _{x \rightarrow 0^{+}}\left(x^{3}\left[2 \psi^{\prime \prime}(x)+x \psi^{\prime \prime \prime}(x)\right]\right)}{\lim _{x \rightarrow 0^{+}}\left(x\left[x \psi^{\prime}(x)-1\right]\right) \lim _{x \rightarrow 0^{+}}\left(x^{2}\left[\psi^{\prime}(x)+x \psi^{\prime \prime}(x)\right]\right)}, \quad x \rightarrow 0^{+} \\
& =-\frac{1}{2} \frac{2}{1 \times(-1)} \\
& =1
\end{aligned}
$$


where we used (5.1), (5.4), and the limit 3.15). In a word, the necessary condition for $H_{\alpha}(x)$ to be completely monotonic on $(0, \infty)$ is $\alpha \geq 2$, while the necessary condition for $-H_{\alpha}(x)$ to be completely monotonic on $(0, \infty)$ is $\alpha \leq 1$.

Using the recurrent formula 3.14 and straightforward computing result in

$$
\begin{aligned}
\mathfrak{H}_{2}(x)-\mathfrak{H}_{2}(x+1)= & 4\left(\frac{1}{x^{2}}+\frac{2}{x}+2\right) \psi^{\prime}(x)-\frac{4 x^{3}+7 x^{2}+6 x+2}{x^{4}} \\
& -\psi^{\prime \prime}(x)-2(2 x+1)\left[\psi^{\prime}(x)\right]^{2} .
\end{aligned}
$$

Let $H_{1}(x)=\mathfrak{H}_{2}(x)-\mathfrak{H}_{2}(x+1)$. Then

$$
H_{1}(x)-H_{1}(x+1)=4\left[\psi^{\prime}(x)\right]^{2}+\frac{\left[\begin{array}{c}
4 x^{6}+20 x^{5}+41 x^{4}+48 x^{3}+37 x^{2}+16 x+4 \\
-4(x+1)^{2}\left(2 x^{3}+5 x^{2}+4 x+2\right) x^{2} \psi^{\prime}(x)
\end{array}\right]}{x^{4}(x+1)^{4}} .
$$

Let $H_{2}(x)=H_{1}(x)-H_{1}(x+1)$. Then

$$
H_{2}(x)-H_{2}(x+1)=\frac{4\left[\psi^{\prime}(x)-\frac{2 x^{4}+13 x^{3}+29 x^{2}+27 x+8}{2 x(x+1)^{2}(x+2)^{2}}\right]}{(x+1)^{2}(x+2)^{2}} .
$$

Let $H_{3}(x)=\frac{(x+1)^{2}(x+2)^{2}}{4}\left[H_{2}(x)-H_{2}(x+1)\right]$. Then, by virtue of 2.1 for $n=1$ and

$$
\frac{1}{x^{r}}=\frac{1}{\Gamma(r)} \int_{0}^{\infty} t^{r-1} e^{-x t} \mathrm{~d} t
$$

in [1, p. $255,6.1 .1]$, we obtain

$$
\begin{aligned}
H_{3}(x) & =\psi^{\prime}(x)-\left[\frac{1}{x}-\frac{1}{2(x+1)}+\frac{1}{2(x+1)^{2}}+\frac{1}{2(x+2)}+\frac{1}{2(x+2)^{2}}\right] \\
& =\int_{0}^{\infty}\left[\frac{t}{1-e^{-t}}-\left(1-\frac{e^{-t}}{2}+\frac{t e^{-t}}{2}+\frac{e^{-2 t}}{2}+\frac{t e^{-2 t}}{2}\right)\right] e^{-x t} \mathrm{~d} t \\
& =\frac{1}{2} \int_{0}^{\infty} \frac{2(t-1) e^{3 t}-(t-3) e^{2 t}-2 e^{t}+t+1}{e^{t}-1} e^{-(x+2) t} \mathrm{~d} t \\
& =\frac{1}{2} \int_{0}^{\infty}\left[\sum_{k=3}^{\infty} \frac{2\left(3 \times 2^{k-2}-1\right)+(k-3)\left(2 \times 3^{k-1}-2^{k-1}\right)}{k !} t^{k}\right] \frac{e^{-(x+2) t}}{e^{t}-1} \mathrm{~d} t .
\end{aligned}
$$

Consequently, the function $H_{3}(x)$, and then the difference $H_{2}(x)-H_{2}(x+1)$, is a completely monotonic function on $(0, \infty)$. Hence, we have

$$
(-1)^{n}\left[H_{2}(x)-H_{2}(x+1)\right]^{(n)}=(-1)^{n}\left[H_{2}(x)\right]^{(n)}-(-1)^{n}\left[H_{2}(x+1)\right]^{(n)} \geq 0
$$

for $n \geq 0$. By induction, it follows that

$$
(-1)^{n}\left[H_{2}(x)\right]^{(n)} \geq(-1)^{n}\left[H_{2}(x+1)\right]^{(n)} \geq(-1)^{n}\left[H_{2}(x+2)\right]^{(n)} \geq \cdots
$$




$$
\geq(-1)^{n}\left[H_{2}(x+m)\right]^{(n)} \geq \cdots \geq \lim _{m \rightarrow \infty}(-1)^{n}\left[H_{2}(x+m)\right]^{(n)} .
$$

It is not difficult to see that

$$
\begin{aligned}
{\left[H_{2}(x)\right]^{(n)}=} & 4\left(\left[\psi^{\prime}(x)\right]^{2}\right)^{(n)}-\left[\frac{4\left(2 x^{3}+5 x^{2}+4 x+2\right)}{x^{2}(x+1)^{2}} \psi^{\prime}(x)\right]^{(n)} \\
& +\left[\frac{4 x^{6}+20 x^{5}+41 x^{4}+48 x^{3}+37 x^{2}+16 x+4}{x^{4}(x+1)^{4}}\right]^{(n)} \\
& \rightarrow 0
\end{aligned}
$$

as $x \rightarrow \infty$ for all $n \geq 0$. Accordingly, we obtain $(-1)^{n}\left[H_{2}(x)\right]^{(n)} \geq 0$ on $(0, \infty)$ for all $n \geq 0$, that is, the function $H_{2}(x)$ is completely monotonic on $(0, \infty)$. Hence, we have

$$
(-1)^{n}\left[H_{1}(x)-H_{1}(x+1)\right]^{(n)}=(-1)^{n}\left[H_{1}(x)\right]-(-1)^{n}\left[H_{1}(x+1)\right]^{(n)} \geq 0
$$

for $n \geq 0$. By induction, it follows that

$$
\begin{aligned}
(-1)^{n}\left[H_{1}(x)\right]^{(n)} \geq & (-1)^{n}\left[H_{1}(x+1)\right]^{(n)} \geq(-1)^{n}\left[H_{1}(x+2)\right]^{(n)} \geq \cdots \\
& \geq(-1)^{n}\left[H_{1}(x+m)\right]^{(n)} \geq \cdots \geq \lim _{m \rightarrow \infty}(-1)^{n}\left[H_{1}(x+m)\right]^{(n)} .
\end{aligned}
$$

It is not difficult to see that

$$
\begin{array}{r}
{\left[H_{1}(x)\right]^{(n)}=4\left[\left(\frac{1}{x^{2}}+\frac{2}{x}+2\right) \psi^{\prime}(x)\right]^{(n)}-\left(\frac{4 x^{3}+7 x^{2}+6 x+2}{x^{4}}\right)^{(n)}} \\
-\left[\psi^{\prime \prime}(x)\right]^{(n)}- \begin{cases}2(2 x+1)\left[\psi^{\prime}(x)\right]^{2} \rightarrow 0, & n=0 \\
2(2 x+1)\left(\left[\psi^{\prime}(x)\right]^{2}\right)^{(n)}+4 n\left(\left[\psi^{\prime}(x)\right]^{2}\right)^{(n-1)} \rightarrow 0, & n \geq 1\end{cases}
\end{array}
$$

as $x \rightarrow \infty$. This means that $(-1)^{n}\left[H_{1}(x)\right]^{(n)} \geq 0$ on $(0, \infty)$ for all $n \geq 0$. In other words,

$$
(-1)^{n}\left[\mathfrak{H}_{2}(x)-\mathfrak{H}_{2}(x+1)\right]^{(n)}=(-1)^{n}\left[\mathfrak{H}_{2}(x)\right]^{(n)}-(-1)^{n}\left[\mathfrak{H}_{2}(x+1)\right]^{(n)} \geq 0
$$

which inductively reduces to

$$
\begin{aligned}
(-1)^{n}\left[\mathfrak{H}_{2}(x)\right]^{(n)} \geq(-1)^{n}\left[\mathfrak{H}_{2}(x+1)\right]^{(n)} \geq(-1)^{n}\left[\mathfrak{H}_{2}(x+2)\right]^{(n)} \geq \cdots \\
\geq(-1)^{n}\left[\mathfrak{H}_{2}(x+m)\right]^{(n)} \geq \cdots \geq \lim _{m \rightarrow \infty}(-1)^{n}\left[\mathfrak{H}_{2}(x+m)\right]^{(n)}
\end{aligned}
$$


for all $n \geq 0$. From the integral representation (2.1) and the formulas (5.1) and $(5.3)$, it is immediate that

$$
\begin{aligned}
{\left[\mathfrak{H}_{2}(x)\right]^{(n)}=} & \psi^{(n+1)}(x)-(-1)^{n} \int_{0}^{\infty} \frac{e^{t}\left(2 e^{t}-t-2\right) t^{n+1}}{\left(e^{t}-1\right)^{2}} e^{-x t} \mathrm{~d} t \\
& +2(-1)^{n} \sum_{k=0}^{n}\left(\begin{array}{l}
n \\
k
\end{array}\right)\left[\int_{0}^{\infty} \frac{e^{t}\left(e^{t}-1-t\right) t^{k}}{\left(e^{t}-1\right)^{2}} e^{-x t} \mathrm{~d} t\right] \\
& \times\left[\int_{0}^{\infty} \frac{e^{t}\left(e^{t}-1-t\right) t^{n-k}}{\left(e^{t}-1\right)^{2}} e^{-x t} \mathrm{~d} t\right] \\
& \rightarrow 0
\end{aligned}
$$

as $x \rightarrow \infty$ for all $n \geq 0$. This means that $(-1)^{n}\left[\mathfrak{H}_{2}(x)\right]^{(n)} \geq 0$ on $(0, \infty)$ for all $n \geq 0$. In other words, the function $\mathfrak{H}_{2}(x)$ is completely monotonic on $(0, \infty)$.

When $\alpha>2$, since

$$
\mathfrak{H}_{\alpha}(x)=\mathfrak{H}_{2}(x)+(\alpha-2)\left[x \psi^{\prime}(x)-1\right]^{2}
$$

and, by virtue of 5.1 ,

$$
x \psi^{\prime}(x)-1=\int_{0}^{\infty} \frac{e^{t}\left(e^{t}-1-t\right)}{\left(e^{t}-1\right)^{2}} e^{-x t} \mathrm{~d} t
$$

is completely monotonic on $(0, \infty)$, from the fact that the product of any finitely many completely monotonic functions is still completely monotonic, it follows that, when $\alpha>2$, the function $\mathfrak{H}_{\alpha}(x)$ is completely monotonic on $(0, \infty)$. As a result, the condition $\alpha \geq 2$ is sufficient for the function $\mathfrak{H}_{\alpha}(x)$ to be completely monotonic on $(0, \infty)$.

The complete monotonicity of $\mathfrak{H}_{2}(x)$ implies $\mathfrak{H}_{2}(x)>0$, which is equivalent to the left hand side of the double inequality $(1.9)$, on $(0, \infty)$.

Employing the recurrent formula (3.14) and direct computing arrive at

$$
\begin{aligned}
\mathfrak{H}_{1}(x+1)-\mathfrak{H}_{1}(x)= & \frac{1}{x^{4}}+\frac{4}{x^{3}}+\frac{4}{x^{2}}+\frac{2}{x}-2\left(\frac{1}{x^{2}}+\frac{2}{x}+2\right) \psi^{\prime}(x) \\
& +(2 x+1)\left[\psi^{\prime}(x)\right]^{2}+\psi^{\prime \prime}(x) .
\end{aligned}
$$

Let $G_{1}(x)=\mathfrak{H}_{1}(x+1)-\mathfrak{H}_{1}(x)$. Then

$$
\begin{aligned}
G_{1}(x)-G_{1}(x+1)= & \frac{2\left(2 x^{3}+5 x^{2}+4 x+2\right)}{x^{2}(x+1)^{2}} \psi^{\prime}(x)-2\left[\psi^{\prime}(x)\right]^{2} \\
& -\frac{2 x^{6}+10 x^{5}+19 x^{4}+22 x^{3}+18 x^{2}+8 x+2}{x^{4}(x+1)^{4}} .
\end{aligned}
$$


Let $G_{2}(x)=G_{1}(x)-G_{1}(x+1)$. Then

$$
\begin{aligned}
G_{2}(x)-G_{2}(x+1)= & \frac{2}{(x+1)^{2}(x+2)^{2}}\left[\frac{4}{x}+\frac{1}{x^{2}}-\frac{1}{x+1}+\frac{1}{2(x+1)^{2}}\right. \\
& \left.+\frac{1}{x+2}+\frac{1}{2(x+2)^{2}}-\psi^{\prime}(x)\right]
\end{aligned}
$$

Let $G_{3}(x)=\frac{(x+1)^{2}(x+2)^{2}}{2}\left[G_{2}(x)-G_{2}(x+1)\right]$. Then, by virtue of 2.1 for $n=1$ and the formula $(5.5)$, we acquire

$$
\begin{aligned}
G_{3}(x) & =\int_{0}^{\infty}\left(4+t-e^{-t}+\frac{t}{2} e^{-t}+e^{-2 t}+\frac{t}{2} e^{-2 t}-\frac{t}{1-e^{-t}}\right) e^{-x t} \mathrm{~d} t \\
& =\frac{1}{2} \int_{0}^{\infty} \frac{8 e^{3 t}-(t+10) e^{2 t}+4 e^{t}-t-2}{e^{t}-1} e^{-(x+2) t} \mathrm{~d} t \\
& =\int_{0}^{\infty}\left[3 t+2 \sum_{k=2}^{\infty} \frac{2^{k+1}\left[\left(\frac{3}{2}\right)^{k}-\frac{k+5}{4}\right]+1}{k !} t^{k}\right] \frac{e^{-(x+2) t}}{e^{t}-1} \mathrm{~d} t \\
& =\int_{0}^{\infty}\left(3 t+8 t^{2}+\frac{32 t^{3}}{3}+\frac{115 t^{4}}{12}+\frac{129 t^{5}}{20}+\frac{139 t^{6}}{40}+\cdots\right) \frac{e^{-(x+2) t}}{e^{t}-1} \mathrm{~d} t .
\end{aligned}
$$

By calculus, we can easily verify that $\left(\frac{3}{2}\right)^{k}-\frac{k+5}{4}>0$ for $k \geq 2$. Therefore, the function $G_{3}(x)$ is completely monotonic on $(0, \infty)$. Hence, the difference $G_{2}(x)-$ $G_{2}(x+1)$ is completely monotonic on $(0, \infty)$. As discussed on the functions $\mathrm{H}_{2}(x)$, $H_{1}(x)$, and $\mathfrak{H}_{2}(x)$ above, we can turn out that the functions $G_{2}(x), G_{1}(x)$, and $-\mathfrak{H}_{1}(x)$ are all completely monotonic on $(0, \infty)$. Furthermore, as discussed on $\mathfrak{H}_{\alpha}$ for $\alpha>2$ above, we can prove that the function $\mathfrak{H}_{\alpha}$ is completely monotonic on $(0, \infty)$ for all $\alpha<1$. Consequently, the condition $\alpha \leq 1$ is sufficient for the function $-\mathfrak{H}_{\alpha}$ to be completely monotonic on $(0, \infty)$.

The complete monotonicity of $-\mathfrak{H}_{1}(x)$ implies $\mathfrak{H}_{1}(x)<0$, which is equivalent to the right hand side of the double inequality $(1.9)$, on $(0, \infty)$.

The sharpness of the double inequality 1.9$)$ on $(0, \infty)$ is concluded from the limits (3.5) and (3.6). The proof of Theorem 4 is complete.

Remark 1 This paper is a revised version of the electronic preprint 17].

Acknowledgements The author is thankful to Alice Le Brigant, the first author of the papers 8.9, for her telling her conjecture on the lower bound $-\frac{1}{2}$ of the sectional curvature $K(x, y)$ and for her supplying related material on 19 February 2020 and later.

The author appreciates anonymous referees for their very careful corrections to and highly valuable comments on the original version of this paper. 


\section{Conflict of interest}

The author declares that he has no conflict of interest.

\section{References}

1. M. Abramowitz and I. A. Stegun (Eds), Handbook of Mathematical Functions with Formulas, Graphs, and Mathematical Tables, National Bureau of Standards, Applied Mathematics Series 55, Reprint of the 1972 edition, Dover Publications, Inc., New York, 1992.

2. H. Alzer and S. Koumandos, Sub-and super-additive properties of Fejér's sine polynomial, Bull. London Math. Soc. 38 (2006), no. 2, 261-268; available online at https://doi.org/ 10.1112/S0024609306018273

3. H. Alzer and S. Ruscheweyh, A subadditive property of the gamma function, J. Math. Anal. Appl. 285 (2003), 564-577; available online at https://doi.org/10.1016/S0022-247X(03) 00425-6

4. H. Alzer and J. Wells, Inequalities for the polygamma functions, SIAM J. Math. Anal. 29 (1998), no. 6, 1459-1466; available online at https://doi.org/10.1137/ S0036141097325071

5. C.-P. Chen, F. Qi, and H. M. Srivastava, Some properties of functions related to the gamma and psi functions, Integral Transforms Spec. Funct. 21 (2010), no. 2, 153-164; available online at https://doi.org/10.1080/10652460903064216

6. B.-N. Guo and F. Qi, A completely monotonic function involving the tri-gamma function and with degree one, Appl. Math. Comput. 218 (2012), no. 19, 9890-9897; available online at https://doi.org/10.1016/j.amc.2012.03.075.

7. B.-N. Guo, F. Qi, and Q.-M. Luo, The additivity of polygamma functions, Filomat 29 (2015), no. 5, 1063-1066; available online at https://doi.org/10.2298/FIL1505063G

8. A. Le Brigant and S. Puechmorel, The Fisher-Rao geometry of beta distributions applied to the study of canonical moments, arXiv preprint (2019), available online at https: //arxiv.org/abs/1904.08247v1.

9. A. Le Brigant, S. Preston, and S. Puechmorel, Fisher-Rao geometry of Dirichlet distributions, arXiv preprint (2020), available online at https://arxiv.org/abs/2005.05608

10. W.-H. Li and F. Qi, Some Hermite-Hadamard type inequalities for functions whose $n$-th derivatives are $(\alpha, m)$-convex, Filomat 27 (2013), no. 8, 1575-1582; available online at https://doi.org/10.2298/FIL1308575L

11. A. W. Marshall, I. Olkin, and B. C. Arnold, Inequalities: Theory of Majorization and its Applications, 2nd Ed., Springer Verlag, New York-Dordrecht-Heidelberg-London, 2011; available online at https://doi.org/10.1007/978-0-387-68276-1

12. D. S. Mitrinović, J. E. Pečarić, and A. M. Fink, Classical and New Inequalities in Analysis, Kluwer Academic Publishers, Dordrecht-Boston-London, 1993; available online at https: //doi.org/10.1007/978-94-017-1043-5

13. C. P. Niculescu and L.-E. Persson, Convex Functions and Their Applications: A Contemporary Approach, Second edition, CMS Books in Mathematics/Ouvrages de Mathématiques de la SMC, Springer, Cham, 2018; available online at https://doi.org/ 10.1007/978-3-319-78337-6

14. F. Qi, A double inequality for the ratio of two non-zero neighbouring Bernoulli numbers, J. Comput. Appl. Math. 351 (2019), 1-5; available online at https://doi.org/10.1016/ j.cam.2018.10.049 
15. F. Qi, Complete monotonicity of a function involving the tri- and tetra-gamma functions, Proc. Jangjeon Math. Soc. 18 (2015), no. 2, 253-264; available online at https://doi. org/10.17777/pjms.2015.18.2.253.

16. F. Qi, Limit formulas for ratios between derivatives of the gamma and digamma functions at their singularities, Filomat 27 (2013), no. 4, 601-604; available online at https://doi. org/10.2298/FIL1304601Q

17. F. Qi, Some properties of several functions involving polygamma functions and originating from the sectional curvature of the beta manifold, HAL preprint (2020), available online at https://hal.archives-ouvertes.fr/hal-02496404

18. F. Qi and R. P. Agarwal, On complete monotonicity for several classes of functions related to ratios of gamma functions, J. Inequal. Appl. 2019, Paper No. 36, 42 pages; available online at https://doi.org/10.1186/s13660-019-1976-z

19. F. Qi and C. Berg, Complete monotonicity of a difference between the exponential and trigamma functions and properties related to a modified Bessel function, Mediterr. J. Math. 10 (2013), no. 4, 1685-1696; available online at https://doi.org/10.1007/ s00009-013-0272-2

20. F. Qi and B.-N. Guo, Complete monotonicity of divided differences of the di- and trigamma functions with applications, Georgian Math. J. 23 (2016), no. 2, 279-291; available online at https://doi.org/10.1515/gmj-2016-0004

21. F. Qi and B.-N. Guo, Necessary and sufficient conditions for functions involving the triand tetra-gamma functions to be completely monotonic, Adv. Appl. Math. 44 (2010), no. 1, 71-83; available online at https://doi.org/10.1016/j.aam.2009.03.003

22. F. Qi and B.-N. Guo, Some properties of extended remainder of Binet's first formula for logarithm of gamma function, Math. Slovaca 60 (2010), no. 4, 461-470; available online at https://doi.org/10.2478/s12175-010-0025-7

23. F. Qi, S. Guo, and B.-N. Guo, Complete monotonicity of some functions involving polygamma functions, J. Comput. Appl. Math. 233 (2010), no. 9, 2149-2160; available online at https://doi.org/10.1016/j.cam.2009.09.044

24. F. Qi and A.-Q. Liu, Completely monotonic degrees for a difference between the logarithmic and psi functions, J. Comput. Appl. Math. 361 (2019), 366-371; available online at https: //doi.org/10.1016/j.cam.2019.05.001.

25. R. L. Schilling, R. Song, and Z. Vondraček, Bernstein Functions, 2nd ed., de Gruyter Studies in Mathematics 37, Walter de Gruyter, Berlin, Germany, 2012; available online at https://doi.org/10.1515/9783110269338

26. D. V. Widder, The Laplace Transform, Princeton University Press, Princeton, 1946.

27. Z.-H. Yang, Some properties of the divided difference of psi and polygamma functions, J. Math. Anal. Appl. 455 (2017), no. 1, 761-777; available online at https://doi.org/10. $1016 / \mathrm{j} \cdot \mathrm{jmaa} .2017 .05 .081$ 